\title{
New Insights Into the Biological Interaction Between Unripe Citrus Fruits and the Tephritid Fly Bactrocera Minax Based on Omics
}

\section{Guijian Zhang}

Huazhong Agricultural University College of Plant Science and Technology

\section{Penghui Xu}

Huazhong Agricultural University College of Plant Science and Technology

\section{Yaohui Wang}

Shanghai Institute of Plant Physiology and Ecology: Institute of Plant Physiology and Ecology Shanghai Institutes for Biological Sciences

\section{Shuai Cao}

Huazhong Agricultural University College of Plant Science and Technology

Xuewei Qi

Huazhong Agricultural University College of Plant Science and Technology

\section{Xueming Ren}

Huazhong Agricultural University College of Plant Science and Technology

ChangYing Niu ( $\square$ niuchangying88@163.com)

College of Plant Science \& Technology, Huazhong Agricultural University

\section{Research Article}

Keywords: citrus fruit, tephritids, interaction, plant secondary metabolite, detoxification genes

Posted Date: July 21 st, 2021

DOl: https://doi.org/10.21203/rs.3.rs-556841/v1

License: (a) (1) This work is licensed under a Creative Commons Attribution 4.0 International License. Read Full License 


\section{Abstract}

The adaptation of phytophagous insects to host defence is an important aspect of plant-insect interactions. The reciprocal adaptability between specialist insects and their hosts have been adequately explored; however, the mechanisms underlying the adaptation of tephritid fruit fly specialists, a group of notorious pests worldwide, to unripen host fruits remain elusive. Here, plant metabolomes and insect transcriptomes were analysed for the first time to explore the interaction between unripe citrus fruits and the Chinese citrus fly Bactrocera minax. Seventeen citrus secondary metabolites, mainly flavones, alkaloids and phenylpropanoids, were identified in the unripe citrus fruit metabolome and they accumulated during larval feeding. Three detoxification genes (1 P450 gene, 2 ABCs genes) were highly expressed in $B$. minax larvae collected from unripe citrus fruits compared with the ones fed on artificial diets and ripe citrus fruits. Based on omics data, a novel $A B C$ gene was screened through plant allelopathy tests and the gene was significantly upregulated in $B$. minax larvae treated with defensive secondary metabolites; additionally, the mortality rate of the larvae reached $51 \%$ after silencing the $A B C$ gene by RNAi technique. Overall, these results shed light on the mechanisms underlying the biological interactions between tephritid fruit fly specialists and host fruits.

\section{Key Message}

- Little was known about the molecular mechanisms underlying the adaptation of $B$. minax to unripe citrus fruits.

- Fifty-four differential expression metabolites were detected in the unripe citrus fruit metabolome and 17 citrus secondary metabolites significantly upregulated during B. minax larval feeding.

- Three detoxification genes were highly expressed in the $B$. minax larvae collected from unripe citrus fruits, and BmOGS12791 knockdown decreased B. minax larval survival rate in unripe citrus fruits.

- This work shed light on the mechanism of the biological interactions between tephritid fruit fly specialists and host fruits.

\section{Introduction}

Herbivorous insects closely interact with their host plants, which provide food resources, oviposition sites and habitat throughout their life cycles (Futuyma et al., 2009). In the long arms race with herbivorous insects, plants have evolved complex defence systems to resist infestation (Kessler et al., 2002; Chuang et al., 2014). Constitutive defence involves physical and chemical defensive traits, such as cuticles and plant secondary metabolites such as nicotine (Wu et al., 2010). The inducible defence of plants occurs after being attacked by herbivorous insects and is attributed to the phenylpropanoid and octadecanoid pathways mediated by salicylic acid (SA) and jasmonic acid (JA), respectively (Hagenbucher et al., 2013). These pathways produce massive plant secondary metabolites to reduce the development and survival of herbivores (Zavala et al., 2004; Howe et al., 2008). For example, the "mustard oil bomb" compound released in brassicaceous plants after herbivorous insect damage exhibits direct toxicity to insects 
and/or acts as a feeding deterrent (Hopkins et al.,2009; Müller et al., 2010; Dixit et al., 2017). Additionally, the concentrations of certain plant secondary metabolites increase in response to herbivory, as reported in cotton, tomato, and coffee, to inhibit herbivorous insect growth and development (Balkema et al., 2003; Magalhães et al., 2008; Bhonwong et al., 2009).

To counteract these plant allelochemicals and other toxic compounds, herbivorous insects have evolved a complete detoxification system, including three important types of detoxifying enzymes (cytochrome P450 monooxygenases, esterases and glutathione $S$-transferases) and two functional gene families, UDP-glucosyltransferases (UGTs) and ATP binding cassette (ABC) transporters (Francis et al., 2005; Jin et al., 2019). These enzyme families have been reported in herbivorous insects such as Aphis gossypii, Helicoverpa armigera, and Spodoptera frugiperda in response to plant secondary metabolites such as gossypol, tannic acid, and nicotine (War et al., 2013; Zou et al., 2016; Li et al., 2019). Enhanced metabolism caused by detoxification genes allows herbivorous insects to survive and complete their development on their host plants. In Myzus persicae nicotianae, the increased expression of $\mathrm{CYP} 6 \mathrm{CY} 3$ and homologous CYP6CY4 genes is related to tolerance to nicotine (Bass et al., 2013). Furthermore, in aphids, some ABC transporters and UGTs showed a dramatic increase in mRNA expression levels after feeding on barley, suggesting that these genes could be critical for detoxification metabolism (Huang et al., 2019).

The Chinese citrus fruit fly Bactrocera minax (Enderlein) (Diptera: Tephritidae) is an oligophagous pest whose host range is almost exclusively restricted to citrus species such as Citrus limon, Citrus aurantium and Citrus sinensis (Wang et al., 2019; Rashid et al., 2021). The female oviposits eggs in green unripe citrus fruits, differing from Bactrocera dorsalis which prefers to oviposit in mature fruits (Zhou et al., 2012; Xu et al., 2019). As an oligophagous insect, B. minax larvae face two challenges: coping with plant secondary metabolites in unripe fruits and completing development within two months. How larvae handle adversity and how citrus fruit metabolites change remain unclear. Previous studies indicated that the adaptation of Rhagoletis pomonella to Rosaceae fruits was most notably related to detoxificationrelated genes such as cytochrome P450s (Ragland et al., 2015). Moreover, the fitness ability of Bactrocera dorsalis is mostly attributed to its complex detoxification system (Shen et al., 2013). Therefore, the identification and analysis of the detoxification enzyme genes of $B$. minax will aid in better understanding the molecular mechanism underlying adaptation to unripe citrus fruits.

The only host of $B$. minax, Citrus spp., is the most produced fruit crop in the world and is cultivated worldwide (Mabberley et al., 2004; Barreca et al., 2011). Citrus fruits are rich dietary sources of flavonoids, which decrease the weight gain of silkworm larvae (Zhang et al., 2012; Dugo et al., 2005). In addition, large amounts of bitter compounds have been detected in citrus fruits, especially limonin and nomilin, and a peak in their contents was observed at the unripe fruit or fruit expansion stage, which corresponds to the B. minax larval development stage (Huang et al., 2019). Aedes albopictus are even killed when exposed to different concentrations of limonids (Hafeez et al., 2011). Additionally, the changes of metabolites in unripe citrus fruits responding to biological stress remains unclear. Exploring 
the composition of metabolites in unripe citrus fruits under biological stress helps to understand the defence mechanism of citrus fruits.

In the current study, we carried out metabolomic analyses of citrus fruits to investigate metabolite changes in response to $B$. minax larvae feeding. Moreover, corresponding B. minax larvae were collected for RNA-Seq to determine the differentially expressed detoxification genes that potentially contribute to host adaptation. These results are expected to provide new insights into the interaction mechanisms between unripe citrus fruits and $B$. minax.

\section{Materials And Methods}

Insects and plants

B. minax larvae and citrus fruits were collected on $31^{\text {st }}$ July, 2018 from San Douping (N 30 $821, \mathrm{E}$ $\left.111^{\circ} 051\right)$, Hubei Province, China. Healthy and active $B$. minax larvae were reared in unripe citrus fruits at $23^{\circ} \mathrm{C}$ in the laboratory.

Citrus fruits with and without infestation by $B$. minax larvae were considered treatment and control samples, respectively. These samples were collected on Sept $1^{\text {st }}$ and Oct $1^{\text {st, }}$ corresponding to $B$. minax first instar larvae and second instar larvae, respectively. The pulp was separated from citrus fruits, immediately placed into liquid nitrogen and then stored at $-80^{\circ} \mathrm{C}$.

RNA isolation, cDNA synthesis and qRT-PCR

Total RNA was isolated using RNA TRIzol (Takara, Japan) following the manufacturer's instructions. First-strand single-strand cDNA was prepared using a PrimerScript ${ }^{\mathrm{TM}} \mathrm{RT}$ Reagent Kit (TaKaRa Bio, Dalian, China) according to the manufacturer's instructions. Samples of $1^{\text {st- }}, 2^{\text {nd- }}$ and $3^{\text {rd }}$-instar larvae were used for stage-specific expression profiles, while different tissues of the $2^{\text {nd }}$-instar larvae were used for tissuespecific expression profiles.

The mRNA levels were measured by quantitative real-time polymerase chain reaction (qRT-PCR) using SYBR® Premix Ex Taq ${ }^{\text {TM }}$ II (Tli RNaseH Plus, TaKaRa Bio) with StepOnePlus ${ }^{\text {TM }}$ (Thermo Fisher Scientific). Real-time PCRs were performed in technical triplicates under the following procedures: a holding cycle of $95^{\circ} \mathrm{C}$ for $30 \mathrm{~s}$, followed by 40 cycling stages of $95^{\circ} \mathrm{C}$ for $5 \mathrm{~s}, 55^{\circ} \mathrm{C}$ for $30 \mathrm{~s}$, and $72^{\circ} \mathrm{C}$ for $31 \mathrm{~s}$. The relative expression was calculated based on the $2^{-\triangle \Delta C T}$ method (Livak and Schmittgen, 2001).

\section{Analysis of citrus fruit metabolomics based on LC-MS data}

The citrus pulp was ground by zirconia beads in a Mixer mill (MM 400) for $1.5 \mathrm{~min}$ at $30 \mathrm{~Hz}$. One hundred milligrams of powder and $1.0 \mathrm{ml}$ of $70 \%$ aqueous methanol were mixed and incubated overnight at $4^{\circ} \mathrm{C}$ for extraction. Before LC-MS analysis, the extracts were absorbed by centrifugation at $10000 \mathrm{~g}$ for $10 \mathrm{~min}$ and filtered through a microporous membrane $(0.22 \mu \mathrm{m}$ pore size $)$. 
Samples $(2 \mu \mathrm{l})$ were injected into an LC-ESI-MS/MS system (HPLC, Shim-pack UFLC SHIMADZU CBM30A), and a column (Waters ACQUITY UPLC HSS T3 C18, $1.8 \mu \mathrm{m}, 2.1 \mu \mathrm{m} * 100 \mathrm{~mm}$ ) was used to analyse the sample extracts. The solvent system included water ( $0.04 \%$ acetic acid) as solution $A$ and acetonitrile $(0.04 \%$ acetic acid) as solution $B$ following the gradient program. The $A: B(v / v)$ gradient was 95:5 at $0 \mathrm{~min}, 5: 95$ at $11.0 \mathrm{~min}, 5: 95$ at $12.0 \mathrm{~min}, 95: 5$ at $12.1 \mathrm{~min}$, and $95: 5$ at $15.0 \mathrm{~min}$. The flow rate was kept at $0.40 \mathrm{~mL} / \mathrm{min}$, and the temperature was maintained at $40^{\circ} \mathrm{C}$.

A triple quadrupole-linear ion trap mass spectrometer (Q Trap), API 4500 QTrap LC/MS/MS system, equipped with an ESI Turbo lon-Spray interface, was used to perform linear ion trap (LIT) and triple quadrupole (QQQ) scans. The ESI source operation parameters were carried out following Chen et al. (2013). In brief, the electrospray ionization temperature was $500^{\circ} \mathrm{C}$, ion spray voltage (IS) was $5500 \mathrm{~V}$, and ion source gas I (GSI), gas II (GSII), and curtain gas (CUR) were set at 55, 60, and 25.0 psi, respectively. Ten and $100 \mu \mathrm{mol} / \mathrm{L}$ polypropylene glycol solutions under QQQ and LIT modes were used for instrument tuning and mass calibration, respectively. QQQ scans were acquired as MRM experiments with collision gas (nitrogen) set to $5 \mathrm{psi}$. DP and CE for individual MRM transitions were performed with further DP and CE optimization. A specific set of MRM transitions was monitored for each period according to the metabolites eluted within this period.

Plant allelochemical feeding assays

Second-instar B. minax larvae were used to detect the expression levels of detoxification genes. Synchronous larvae were fed artificial diets supplemented with 6 plant secondary metabolites. The control larvae were fed an artificial diet with the same amount of DMSO $/ \mathrm{H}_{2} \mathrm{O}$. Twenty larvae were fed an artificial diet containing compounds. After $72 \mathrm{~h}$, active larvae were collected for RNA extraction and qRTPCR.

dsRNA preparation and microinjection

dsRNA was synthesized by a Transcript Aid T7 High Yield Transcription Kit (Thermo). The dsRNA DNA template (Table S1) was amplified by primers containing the T7 RNA polymerase promoter at both ends, and the purified DNA template $(1 \mu \mathrm{g})$ was used to synthesize dsRNA. The quality and size of dsRNA were verified by $1 \%$ agarose gel electrophoresis and spectrophotometer (Thermo). Approximately $2 \mu \mathrm{g}$ dsRNA was injected into the abdomens of the second instar larvae by the microinjection method. After injection, the $B$. minax larvae were placed in green citrus, which remained consistent with the natural environment. The survival rate was statistically significant after $72 \mathrm{~h}$, and lively larvae were collected to detect RNAi efficiency. This experiment was replicated 6 times.

\section{Statistical analysis}

SPSS 22.0 and R software were used for statistical analysis. The heat maps and Venn diagrams of the transcriptome and metabolome were examined with an online $\mathrm{R}$ package (http://www.ehbio.com/ImageGP/index.php/Home/Index/PHeatmap.html). The gene expression level 
and survival rate were analysed with SPSS 22.0 software by independent Student's t-test. The data are presented as the means \pm SEM, and significance levels were set at $* P<0.05,{ }^{\star} * P<0.01$, and ${ }^{\star \star \star} P<0.001$.

\section{Results}

\section{Metabolite differences in unripe citrus fruits}

A total of 820 metabolites were detected and quantified in four samples of citrus fruits. By mapping on the Metware database (MWDB), these metabolites were divided into seven classes, of which plant secondary metabolites were the most prevalent ( $62.8 \%$ of the metabolites), followed by amino acids (11.9\%) and lipids (8\%) (Fig 1).

A comparison was performed to screen the metabolites involved in the citrus fruit defensive response to infection by $1^{\text {st }}$ and $2^{\text {nd }}$-instar B. minax larvae (Fig 2a, Fig S1). The results indicated that 105 and 236 metabolites were changed after $1^{\text {st }}$ - and $2^{\text {nd }}$-instar larval feeding, including 88 and 196 upregulated metabolites, respectively (Fig 2b). Among these changed metabolites, plant secondary metabolites accounted for a substantial proportion (69.5\% and $72.5 \%)$. Organic acids, phenylpropanoids and flavones were the main components in $1^{\text {st }}$-instar $B$. minax larvae damaging citrus fruits, followed by terpenes and alkaloids. More plant secondary metabolites were detected in citrus fruits infected by $2^{\text {nd }}$-instar B. minax larvae, in which flavones and organic acids accounted for a substantial proportion, approximately $19.4 \%$ and $15.2 \%$, followed by phenylpropanoids at $11.4 \%$ and alkaloids at $7.2 \%$ (Fig S2).

Fifty-four metabolites were detected to be significantly altered during infection of both $1^{\text {st }}$-and $2^{\text {nd }}$-instar B. minax larvae, of which plant secondary metabolites accounted for $68.5 \%$, including phenylpropanoids, flavones, alkaloids, terpenes and organic acids (Fig 2c, Tab 1). Most metabolites were upregulated in 54 metabolites, and plant secondary metabolites still accounted for a large proportion (65\%). In particular, some metabolites, such as coumaraldehyde and N-methylcytisine, had weak or no signals because of their low contents; however, the signals were obviously enhanced after damage from B. minax larvae (Tab 1). Based on the KEGG analysis, 18 functional pathways were annotated, with most of these compounds concentrated on biosynthesis of secondary metabolites and metabolic pathways (Fig S3).

\section{Differential expression of detoxification genes}

A total of 61 P450s, 17 GSTs, 33 CarEs, 16 UGTs and 46 ABCs were identified to be expressed in the transcriptome of $B$. minax from unripe citrus fruits, with a substantial proportion of genes in these detoxification families being highly expressed in $B$. minax $1^{\text {st }}$ - and $2^{\text {nd }}$-instar larvae, suggesting that these genes might play an important role in the larval development of $B$. minax (Fig 3a). In comparison with those of control larvae reared in unripe citrus fruits, the DEGs in detoxification families were screened to explore the adaptative mechanisms of $B$. minax larvae (date unpublished). Four comparison groups of 
$1^{\text {st }}$ - and $2^{\text {nd }}$-larval transcriptomes were compared. Three detoxification genes ( 1 P450, 2 ABCs) were highly expressed in control group larvae in common compared to both treatment groups (Fig 3b-c, Tab 2).

\section{Induced expression of DEGs by plant allelochemical and expression patterns}

Based on the analysis of citrus fruit metabolism, 6 plant secondary metabolites were selected. NMethylcytisine, tryptamine and coixol are alkaloids that were upregulated after $B$. minax larval damage. The qRT-PCR results indicate that the mRNA expression levels of 6 upregulated detoxification genes were differentially induced when $B$. minax larvae were fed on 6 plant allelochemicals. Interestingly, the expression of BmOGS6416 and BmOGSO653 in B. minax larvae was significantly increased after Nmethylcytisine and nomilin treatment, respectively, which implied the potential interaction between these two metabolites and detoxification genes (Fig 4a). Moreover, a gene, BmOGS12791, belonging to the ABC transporter family, was simultaneously significantly increased in 6 plant allelochemical treatments $(P<$ 0.05). Based on the results above, the expression patterns in different developmental stages and different tissues were determined via qRT-PCR. The results showed that BmOGS12791 exhibited higher expression levels in the $1^{\text {st }}$ - and $2^{\text {nd }}$-instar larvae (Fig $4 \mathrm{~b}$ ) and in the midgut and malpighian tubule (Fig 4c).

\section{Knockdown of BmOGS12791and phenotypic effects}

Microinjection was applied for RNA interference, as shown in Fig 5a. The transcript level of BmOGS12791 decreased by $50 \%$ compared to the control level after $72 \mathrm{~h}$ of injection of $1^{\text {st }}$-instar $B$. minax larvae $(P<0.05)$. The survival rate was also determined after RNAi, and a significant decrease was found in the $d s B m O G S 12791$-treated B. minax larvae compared to that of the dsEGFP-treated larvae (Fig $5 b)$.

\section{Discussion}

Plant-insect interactions are key for their coevolution (Futuyma et al., 2009). In nature, most herbivorous insects are specialists that are closely related to their host plants (Clarke, 2017). To unravel the molecular mechanisms underpinning these interactions, a combined analysis of plant metabolism and the insect transcriptome was performed to explore the interaction between unripe citrus fruits and B. minax larvae. Seventeen secondary metabolites were detected significantly upregulated in the unripe citrus fruit during $B$. minax larval feeding. Meanwhile, a novel $A B C$ gene was screened through plant allelopathy tests which significantly reduced the survival rate of $B$. minax larvae in unripe citrus fruits after RNAi. Understanding how B.minax larvae adapt to host citrus fruits is crucial for exploring the evolution of specialized diets.

Plants produce hundreds of thousands of different specialized metabolites, which function as defences of plants, for example, nicotine in tobacco, gossypol in cotton, and mustard oil glycoside-black mustard enzyme in cruciferous crops (War et al., 2013; Zou et al., 2016; Li et al., 2019). In this study, we found many secondary metabolites in unripe citrus fruits by comparative metabolomics analysis. The 
metabolite changes well explained the phenotypes when infested citrus fruits turned colour from green to yellow and then dropped to the ground before ripening. To date, few studies have focused on the response of unripe citrus fruits to biotic stress, with studies so far only focusing on citrus huanglongbing (Ferrarezi et al., 2020). Therefore, these differentially accumulated plant secondary metabolites identified herein may serve as important biochemical markers for induced resistance against insect herbivores. The plant secondary metabolites mainly contained phenylpropanoids, terpenoids and alkaloids, which were reported to be toxic in Pieris rapae, Schizaphis graminum, and Ashbya gossypii in a previous study (Hagenbucher et al., 2013; Fernandes et al., 2009; Yuling et al., 2019; Wang et al., 2018). Our results implied that these plant secondary metabolites were vitally important for unripe citrus fruit defence against $B$. minax larvae. Similarly, research on the interactions among Anastrepha acris and its highly toxic host plant Hippomane mancinella indicated that phenylpropanoids, flavonoids, chalcones and coumarins induced defence responses (Aluja et al., 2020). Taken together, these findings open a window for further study on the induced defence mechanisms of unripe citrus fruits.

Defence traits have been thought to be acquired by plants at the expense of other plant functions, such as growth and reproduction (Züst et al., 2017). Plant metabolism is usually reprogrammed to enhance specialized metabolism to ward off invaders, while primary metabolism is often suppressed (Zhao et al., 2020). For example, brown planthopper (BPH) infestation of rice upregulated the defensive response but downregulated primary metabolism (Kang et al., 2019). However, in this study, we found that $B$. minax larvae developed significantly with an increase in primary metabolites, including amino acids and vitamins, which may contribute to nutrition for the growth of larvae in unripe citrus fruits. More research is needed to explore the mechanisms by which larvae utilize these metabolites.

The detoxification system plays an important role in the host adaptation of phytophagous insects (Li et al., 2017; Ma et al., 2019). In this study, many differentially expressed detoxification genes belonging to the P450, GST, CarEs, ABC and UGT families were identified in the four comparison groups. Among them, a novel $A B C$ transporter, BmOGS12791, decreased the survival of $B$. minax larvae in unripe citrus after RNA interference, which implied that BmOGS12791 may play a critical role in detoxification and adaptation in B. minax. Moreover, BmOGS12791 exhibited higher expression in the midgut and malpighian tubule, which are universally known to be important tissues associated with the metabolism of xenobiotics (Mao et al., 2007). Notably, ABC transporters have important roles in xenobiotic detoxification and Bt resistance (Wu et al., 2019). The expression of HaABCG11 was upregulated after larvae were fed a diet supplemented with nicotine, and $\mathrm{H} A A B C B 3$ showed higher expression levels in the guts of Helicoverpa armigera larvae after they were fed a diet containing nicotine or tomatine (Huang et al., 2015; Bretschneider et al., 2016). ABCC genes were reported to be associated with resistance to Cry toxins from Bacillus thuringiensis (Bt) by reducing the binding affinity of Cry toxins to brush border membrane vesicles in different lepidopteran species (Pardol et al., 2013; Xiao et al., 2014; Chen et al., 2018). The effects of these detoxification enzymes on the metabolic ability of secondary metabolites and whether they affect the host adaptability of B. minax larvae are worth further study. 
The interaction between $B$. minax larvae and unripe citrus fruits involves many metabolites and genotypic changes. Through the change in the number of differentially abundant metabolites, we speculated that the defence response of unripe citrus fruits gradually increased after the feeding of $B$. minax larvae, which was mainly due to the greater wound on citrus with the growth of larvae. However, $B$. minax larvae had access to nutrients from unripe citrus fruits and completed development within two months (Wang et al., 2019). There must be a specific gene expression system in B. minax larvae that contributes to their adaptation to and utilization of complex citrus metabolites as the adaptation of Plutella xylostella to crucifer (Sun et al., 2009). Here, we only explored the important role of the detoxification system in adapting to citrus, and future studies on the chemoreception system and digestion system in B. minax could better explain the host adaptability of the larvae. On the other hand, exploring the gene transcription level changes of unripe citrus fruits after larval infestation, especially jasmonic acid- and salicylic acidrelated pathways based on transcriptomics, will comprehensively clarify the defence response of unripe citrus fruit upon feeding by $B$. minax larvae.

Overall, the results of current study are discussed with special emphasis on citrus fruits defenceresponsive metabolites induced by $B$. minax larvae and the detoxification genes of $B$. minax involved in the response to citrus defence. Seventeen citrus secondary metabolites and three $B$. minax larval detoxification genes were screened to participate in the biological interaction between unripe citrus fruits and B. minax larvae. By linking the results of citrus metabolism and B. minax larval detoxification genes, we will better understand how coevolution between unripe citrus fruits and $B$. minax larvae shapes specialized diets traits. Moreover, we have screened a key detoxification gene that affects the $B$. minax larval adaptation to the unripe citrus fruits. However, the screening of specific defensive secondary metabolites in citrus fruits is still insufficient, which is the focus of further work. These studies will provide scientific basis for integrated management of $B$. minax.

\section{Declarations}

\section{Acknowledgments}

This study was funded by the Natural Science Foundation of China $(31661143045,31972270)$, and the Joint programme of the Israel Science Foundation and the Science Foundation of China (2482/16). We also would like to acknowledge Dr. Peng Han for critical reading and suggestions for improvement of the manuscript.

\section{Data availability}

The datasets generated during the current study are available from the corresponding author on reasonable request.

\section{Compliance with ethical standards}

\section{Conflict of interest}


The authors declare that they have no conflict of interest.

\section{Ethical approval}

This article does not contain any studies with human participants or animals performed by any of the authors.

\section{Supporting information}

Supporting information may be found in the online version of this article.

\section{References}

1. Aluja M, Pascacio-Villafán C, Altúzar-Molina A, et al (2020) Insights into the Interaction between the Monophagous Tephritid Fly Anastrepha acris and its Highly Toxic Host Hippomane mancinella (Euphorbiaceae). J Chem Ecol 46: 430-441. https://doi.org/10.1007/s10886-020-01164-8

2. Balkema-Boomstra AG, Zijlstra S, Verstappen FWA, et al (2003) Role of cucurbitacin C in resistance to spider mite (Tetranychus urticae) in cucumber (Cucumis sativus L.). J Chem Ecol 29: 225-235. https://doi.org/10.1023/A:1021945101308

3. Barreca D, Bellocco E, Caristi C, et al (2011) Elucidation of the flavonoid and furocoumarin composition and radical-scavenging activity of green and ripe chinotto (Citrus myrtifolia Raf.) fruit tissues, leaves and seeds. Food Chem 129: 1504-1512. https://doi.org/10.1016/j.foodchem.2011.05.130

4. Bass C, Zimmer CT, Riveron JM, et al (2013) Gene amplification and microsatellite polymorphism underlie a recent insect host shift. Proc Natl Acad Sci U S A 110: 19460-19465. https://doi.org/10.1073/pnas.1314122110

5. Bhonwong A, Stout MJ, Attajarusit J, Tantasawat P (2009) Defensive role of tomato polyphenol oxidases against cotton bollworm Helicoverpa armigera and beet armyworm Spodoptera exigua. J Chem Ecol 35: 28-38. https://doi.org/10.1007/s10886-008-9571-7

6. Bretschneider A, Heckel DG, Vogel H (2016) Know your ABCs: Characterization and gene expression dynamics of $A B C$ transporters in the polyphagous herbivore Helicoverpa armigera. Insect Biochem Mol Biol 72: 1-9. https://doi.org/10.1016/j.ibmb.2016.03.001

7. Chen L, Wei J, Liu C, et al (2018) Specific binding protein $A B C C 1$ is associated with Cry2Ab toxicity in Helicoverpa armigera. Front Physiol 9: 1-11. https://doi.org/10.3389/fphys.2018.00745

8. Chuang WP, Ray S, Acevedo FE, et al (2014) Herbivore cues from the fall armyworm (Spodoptera frugiperda) larvae trigger direct defenses in maize. Mol Plant-Microbe Interact 27: 461-470. https://doi.org/10.1094/MPMI-07-13-0193-R

9. Clarke AR (2017) Why so many polyphagous fruit flies (Diptera: Tephritidae)? A further contribution to the "generalism" debate. Biol J Linn Soc 120: 245-257. https://doi.org/10.1111/bij.12880 
10. Dixit G, Praveen A, Tripathi T, et al (2017) Herbivore-responsive cotton phenolics and their impact on insect performance and biochemistry. J ASIA PAC ENTOMOL, 20(2), 341-

351. https://doi.org/10.1016/j.aspen.2017.02.002

11. Dugo P, Presti M Lo, Öhman M, et al (2005) Determination of flavonoids in citrus juices by microHPLC-ESI/MS. J Sep Sci 28: 1149-1156. https://doi.org/10.1002/jssc.200500053

12. Fernandes F, Pereira DM, de Pinho PG, et al (2009) Metabolic fate of dietary volatile compounds in Pieris brassicae. Microchem J 93: 99-109. https://doi.org/10.1016/j.microc.2009.05.006

13. Ferrarezi RS, Vincent $\mathrm{Cl}$, Urbaneja A, Machado MA (2020) Editorial: Unravelling Citrus Huanglongbing Disease. Front Plant Sci 11: 10-12. https://doi.org/10.3389/fpls.2020.609655

14. Francis F, Vanhaelen N, Haubruge E (2005) Glutathione S-transferases in the adaptation to plant secondary metabolites in the Myzus persicae aphid. Arch Insect Biochem Physiol 58: 166-174. https://doi.org/10.1002/arch.20049

15. Futuyma DJ, Agrawal AA (2009) Macroevolution and the biological diversity of plants and herbivores[J]. P Natl Acad Sci USA, 2009, 106(43): 18054-18061. http://doi.org/10.1073/pnas.0904106106

16. Hafeez F, Akram W, Shaalan EAS (2011) Mosquito larvicidal activity of citrus limonoids against Aedes albopictus. Parasitol Res 109: 221-229. https://doi.org/10.1007/s00436-010-2228-9

17. Hagenbucher S, Olson DM, Ruberson JR, et al (2013) Resistance Mechanisms Against Arthropod Herbivores in Cotton and Their Interactions with Natural Enemies. CRC Crit Rev Plant Sci 32: 458482. https://doi.org/10.1080/07352689.2013.809293

18. Hopkins RJ, Van Dam NM, Van Loon JJA (2009) Role of glucosinolates in insect-plant relationships and multitrophic interactions. Annu Rev Entomol 54: 57-83.

https://doi.org/10.1146/annurev.ento.54.110807.090623

19. Howe GA, Jander G (2008) Plant immunity to insect herbivores. Annu Rev Plant Biol 59: 41-66. https://doi.org/10.1146/annurev.arplant.59.032607.092825

20. Huang S, Liu X, Xiong B, et al (2019) Variation in limonin and nomilin content in citrus fruits of eight varieties determined by modified HPLC. Food Sci Biotechnol 28: 641-647. https://doi.org/10.1007/s10068-018-0509-8

21. Huang X, Liu D, Zhang R, Shi X (2019) Transcriptional Responses in Defense-Related Genes of Sitobion avenae (Hemiptera: Aphididae) Feeding on Wheat and Barley. J Econ Entomol 112:382395. https://doi.org/10.1093/jee/toy329

22. Huang XZ, Chen JY, Xiao HJ, et al (2015) Dynamic transcriptome analysis and volatile profiling of Gossypium hirsutum in response to the cotton bollworm Helicoverpa armigera. Sci Rep 5:1-14. https://doi.org/10.1038/srep11867

23. Jin R, Mao K, Liao X, et al (2019) Overexpression of CYP6ER1 associated with clothianidin resistance in Nilaparvata lugens (Stål). Pestic Biochem Physiol 154:39-45.

https://doi.org/10.1016/j.pestbp.2018.12.008

Page $11 / 19$ 
24. Kang K, Yue L, Xia X, et al (2019) Comparative metabolomics analysis of different resistant rice varieties in response to the brown planthopper Nilaparvata lugens Hemiptera: Delphacidae. Metabolomics 15:1-13. https://doi.org/10.1007/s11306-019-1523-4

25. Kessler A, Baldwin IT (2002) Plant responses to insect herbivory: The emerging molecular analysis. Annu Rev Plant Biol 53: 299-328. https://doi.org/10.1146/annurev.arplant.53.100301.135207

26. Li F, Ma K, Chen X, Zhou JJ, et al (2019) The regulation of three new members of the cytochrome P450 CYP6 family and their promoters in the cotton aphid Aphis gossypii by plant allelochemicals. Pest Manag Sci 75(1): 152-9. https://doi.org/doi:10.1002/ps.5081

27. Li F, Ma KS, Liang PZ, et al (2017) Transcriptional responses of detoxification genes to four plant allelochemicals in Aphis gossypii. J Econ Entomol 110: 624-631. https://doi.org/10.1093/jee/tow322

28. Ma K, Li F, Tang Q, et al (2019) CYP4CJ1-mediated gossypol and tannic acid tolerance in Aphis gossypii Glover. Chemosphere 219: 961-970. https://doi.org/10.1016/j.chemosphere.2018.12.025

29. Mabberley DJ (2004) Citrus (Rutaceae): A review of recent advances in etymology, systematics and medical applications. Blumea J Plant Taxon Plant Geogr 49: 481-498. https://doi.org/10.3767/000651904X484432

30. Magalhães STV, Guedes RNC, Demuner AJ, Lima ER (2008) Effect of coffee alkaloids and phenolics on egg-laying by the coffee leaf miner Leucoptera coffeella. Bull Entomol Res 98:483-489. https://doi.org/10.1017/S0007485308005804

31. Mao YB, Cai WJ, Wang JW, et al (2007) Silencing a cotton bollworm P450 monooxygenase gene by plant-mediated RNAi impairs larval tolerance of gossypol. Nat Biotechnol 25: 1307-1313. https://doi.org/10.1038/nbt1352

32. Müller R, Vos M De, Sun JY, Jander G (2010) Differential Effects of Indole and Aliphatic Glucosinolates on Lepidopteran Herbivores. Journal of Chemical Ecology, 2010, 36(8): 905-913. https://doi.org/10.1007/s10886-010-9825-z

33. Pardo-López L, Soberón M, Bravo A (2013) Bacillus thuringiensis insecticidal three-domain Cry toxins: Mode of action, insect resistance and consequences for crop protection. FEMS Microbiol Rev 37: 3-22. https://doi.org/10.1111/j.1574-6976.2012.00341.x

34. Ragland GJ, Almskaar K, Vertacnik KL, et al (2015) Differences in performance and transcriptomewide gene expression associated with Rhagoletis (Diptera: Tephritidae) larvae feeding in alternate host fruit environments. Mol Ecol 24: 2759-2776. https://doi.org/10.1111/mec.13191

35. Rashid M A, Dong Y, Andongma A A, et al (2021) The Chinese Citrus Fly, Bactrocera minax (Diptera: Tephritidae): A Review of its Biology, Behaviour and Area-Wide Management[M]// Area-Wide Integrated Pest Management.

36. Shen GM, Dou W, Huang Y, et al (2013) In silico cloning and annotation of genes involved in the digestion, detoxification and RNA interference mechanism in the midgut of Bactrocera dorsalis [Hendel (Diptera: Tephritidae)]. Insect Mol Biol 22: 354-365. https://doi.org/10.1111/imb.12026 
37. Sun JY, Sønderby IE, Halkier BA, et al (2009) Non-volatile intact indole glucosinolates are host recognition cues for ovipositing Plutella xylostella. J Chem Ecol 35: 1427-1436. https://doi.org/10.1007/s10886-009-9723-4

38. Wang D, Xie N, Yi S, et al (2018) Bioassay-guided isolation of potent aphicidal Erythrina alkaloids against Aphis gossypii from the seed of Erythrina crista-galli L[J]. Pest Manag Sci 74(1): 210-218. https://doi.org/doi: 10.1002/ps.4698

39. Wang Y, Andongma AA, Dong Y, et al (2019) Rh6 gene modulates the visual mechanism of host utilization in fruit fly Bactrocera minax. Pest Manag Sci 75: 1621-1629. https://doi.org/10.1002/ps.5278

40. War AR, Paulraj MG, Hussain B, et al (2013) Effect of plant secondary metabolites on legume pod borer, Helicoverpa armigera. J Pest Sci 86: 399-408. https://doi.org/10.1007/s10340-013-0485-y

41. Wu C, Chakrabarty S, Jin M, et al (2019). Insect ATP-Binding Cassette (ABC) Transporters: Roles in Xenobiotic Detoxification and Bt Insecticidal Activity. Int J Mol Sci. 20(11): 28-29. https://doi.org/doi:10.3390/ijms20112829

42. Wu J, Baldwin IT (2010) New insights into plant responses to the attack from insect herbivores. Annu Rev Genet 44: 1-24. https://doi.org/10.1146/annurev-genet-102209-163500

43. Xiao Y, Zhang T, Liu C, et al (2014) Mis-splicing of the $A B C C 2$ gene linked with Bt toxin resistance in Helicoverpa Armigera. Sci Rep 4:1-7. https://doi.org/10.1038/srep06184

44. Xu P, Wang Y, Akami M, Niu CY (2019) Identification of olfactory genes and functional analysis of BminCSP and BminOBP21 in Bactrocera minax. PLoS One 14: 1-13. https://doi.org/10.1371/journal.pone.0222193

45. Yuling W, Zhijie G, Guanfang H U, et al (2019) Separation, identification and pesticidal activity of alkaloids from Anisodus tanguticus (Maxim.) Pascher[J]. Plant Protection, 2019,45(04):190194+204. (In Chinese)

46. Zavala JA, Patankar AG, Gase K, Baldwin IT (2004) Constitutive and inducible trypsin proteinase inhibitor production incurs large fitness costs in Nicotiana attenuata. Proc Natl Acad Sci USA 101:1607-1612. https://doi.org/10.1073/pnas.0305096101

47. Zhang YE, Ma HJ, Feng DD, et al (2012) Induction of detoxification enzymes by quercetin in the silkworm. J Econ Entomol 105:1034-1042. https://doi.org/10.1603/EC11287

48. Zhao X, Chen S, Wang S, et al (2020) Defensive Responses of Tea Plants (Camellia sinensis) Against Tea Green Leafhopper Attack: A Multi-Omics Study. Front Plant Sci, 10:1-17. https://doi.org/10.3389/fpls.2019.01705

49. Zhou XW, Niu CY, Han P, Desneux N (2012) Field evaluation of attractive lures for the fruit fly Bactrocera minax (diptera: Tephritidae) and their potential use in spot sprays in hubei province (China). J Econ Entomol 105:1277-1284. https://doi.org/10.1603/EC12020

50. Zou X, Xu Z, Zou H, et al (2016) Glutathione S-transferase SIGSTE1 in Spodoptera litura may be associated with feeding adaptation of host plants. Insect Biochem Mol Biol 70:32-43. https://doi.org/10.1016/j.ibmb.2015.10.005

Page 13/19 
51. Züst T, Agrawal AA (2017) Trade-Offs Between Plant Growth and Defense Against Insect Herbivory: An Emerging Mechanistic Synthesis. Annu Rev Plant Biol 68:513-534. https://doi.org/10.1146/annurev-arplant-042916-040856

\section{Tables}

Due to technical limitations, Table 1 is only available as a download in the Supplemental Files section.

Table 2 Detoxification genes in the Bactrocera minax with reference to the detoxification genes present in the Drosophila melanogastergenome

\begin{tabular}{|llll|}
\hline Genome ID & $\boldsymbol{P}$ value & Fly base ID & Best hit \\
\hline Bm0GS02200 & 0 & FBgn0015033 & Cyp4d8-PA \\
\hline Bm0GS03787 & 0 & FBgn0030672 & CG9281-PB \\
\hline Bm0GS12791 & $3.07 E-25$ & FBgn0039890 & CG2316-PA \\
\hline
\end{tabular}

\section{Figures}
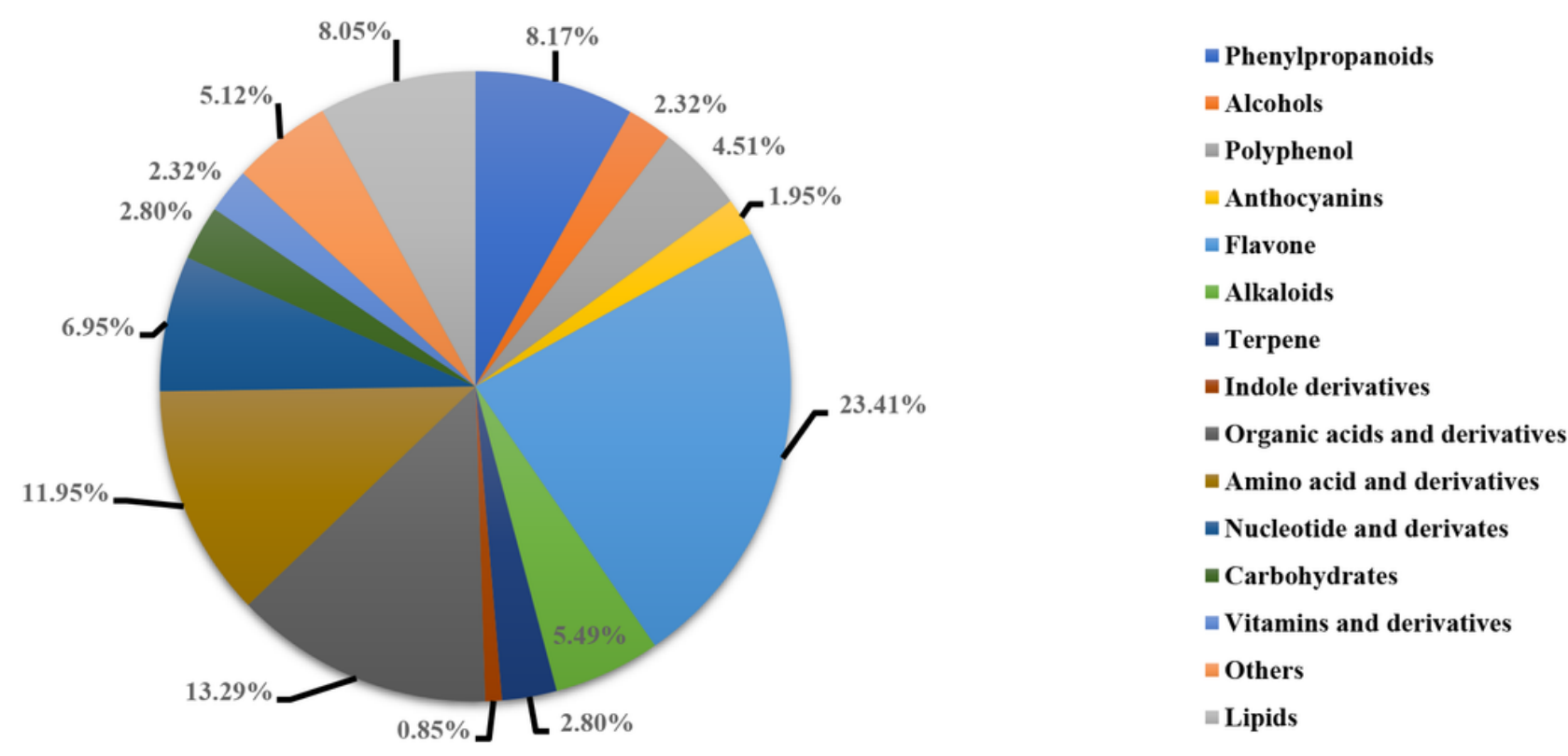

Figure 1

Pie chart analysis of the metabolite composition of unripe citrus fruits. 
a

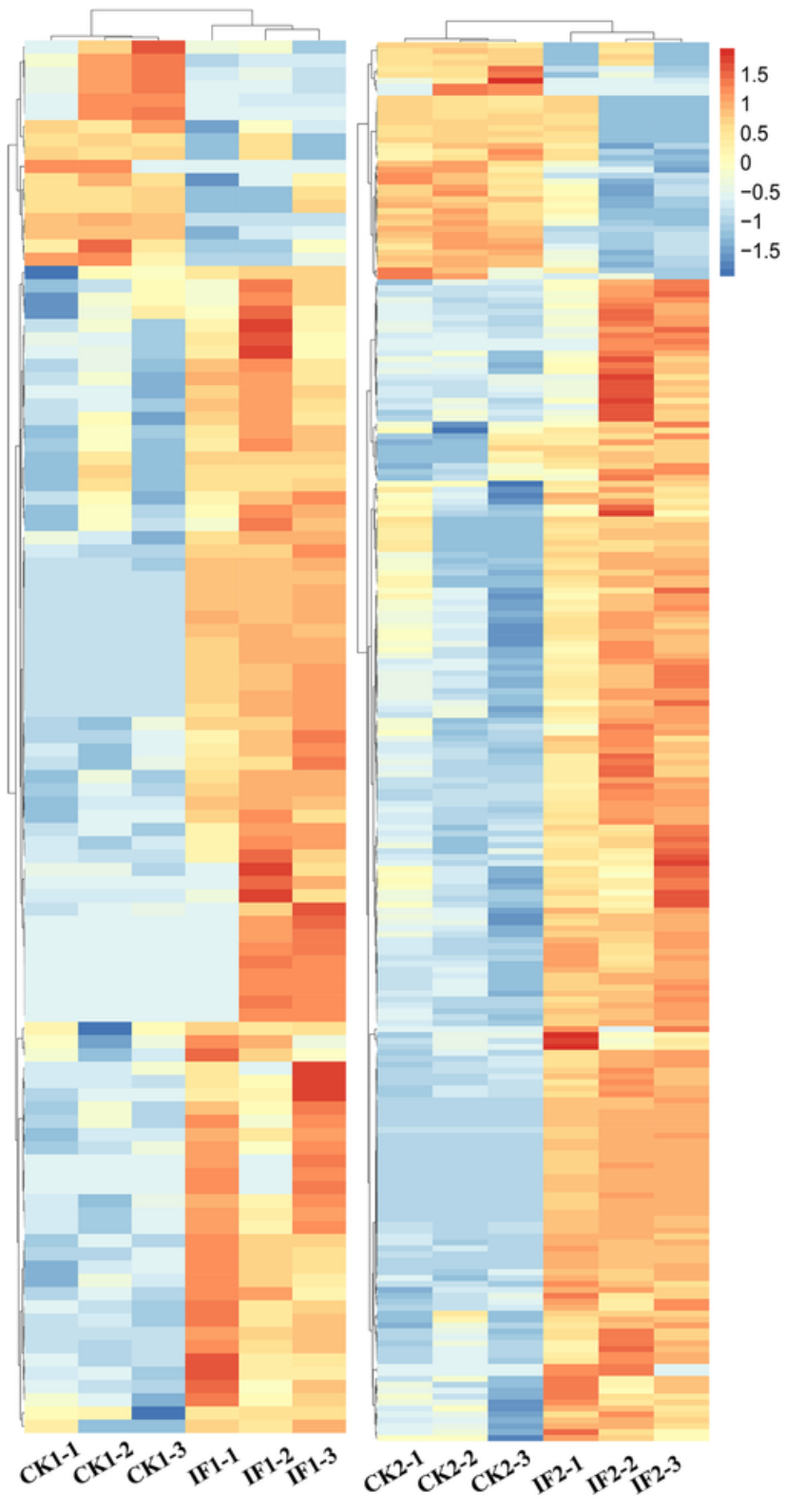

b

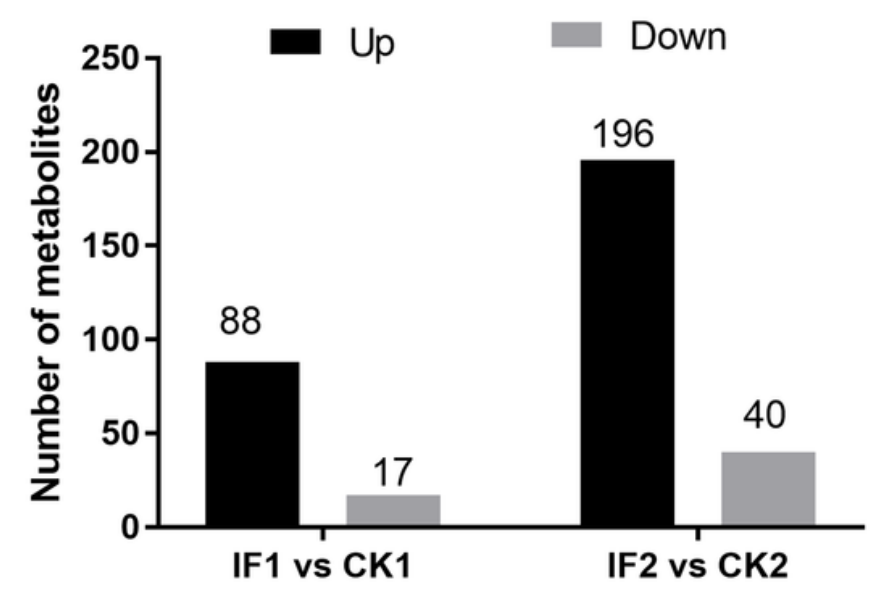

C

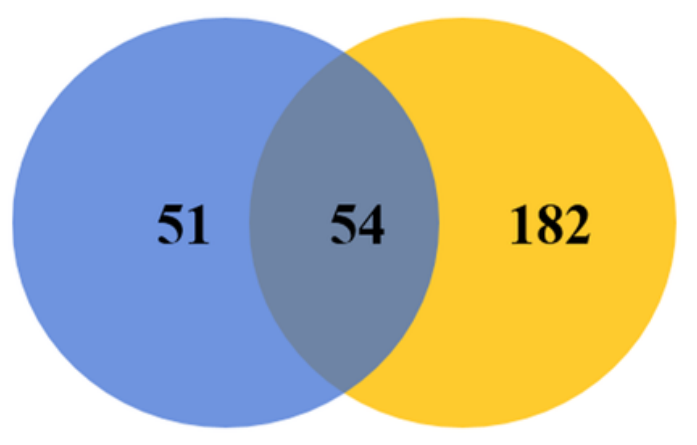

IF1 VS CK1

IF2 VS CK2

\section{Figure 2}

Analysis of differential metabolites in citrus fruits in response to B. minax larval feeding. a: Heatmap of differential metabolites in citrus fruits. IF, Citrus fruits infected by $1 \mathrm{st}$ and 2 nd instar B. minax larvae. CK, natural citrus fruits without $B$. minax larval feeding. $b$ : Distribution of upregulated and downregulated metabolites of citrus fruits exposed to 1 st- and 2 nd-instar larval feeding in each comparison; c: Venn diagram analysis of DEGs in different comparisons. 

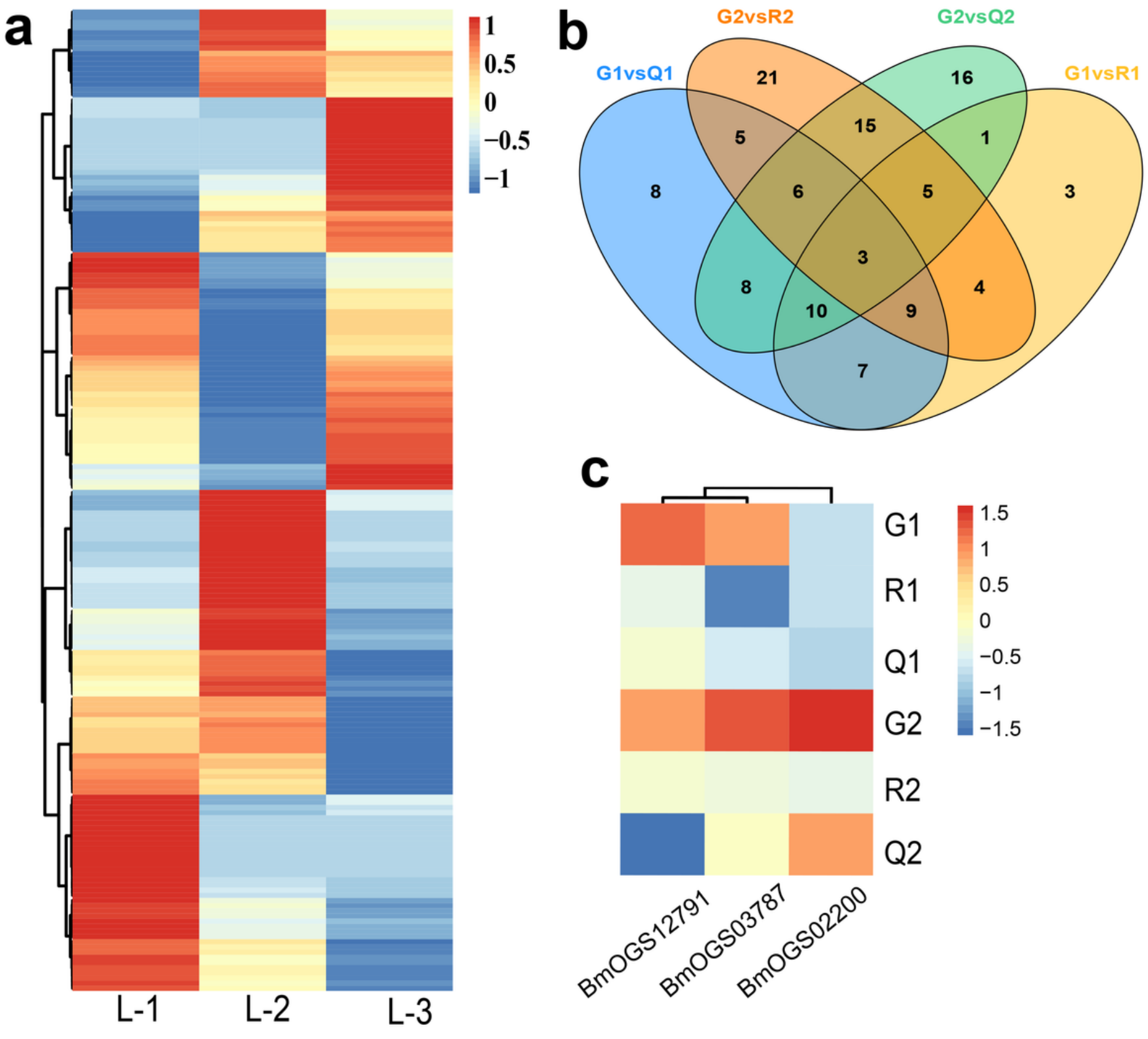

\section{R2}

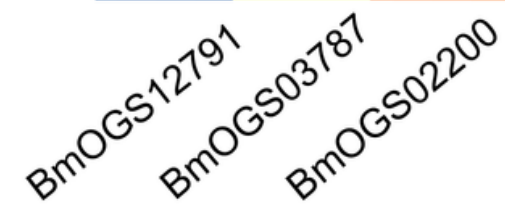

Q2

\section{Figure 3}

Analysis of differentially expressed detoxification genes in B. minax larvae. a: Heatmap of detoxification genes in 1st-, 2nd- and 3rd- instar larvae using an average value of three replicates. b: Venn diagram analysis of DEGs in different comparisons among groups. G, Larvae reared in green citrus fruits; $R$, Larvae reared in ripe citrus fruits; Q, Larvae rearing on an artificial diet. c: Heatmap of 3 detoxification genes highly expressed in B. minax larvae reared in green citrus fruits. 
a

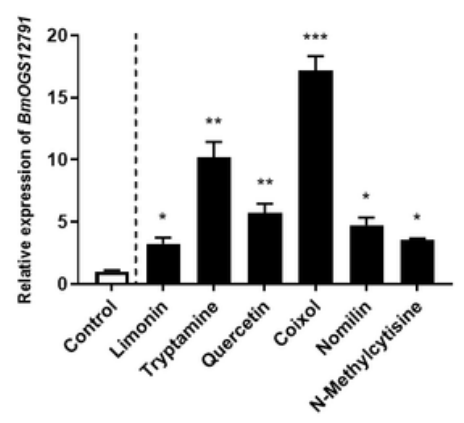

b

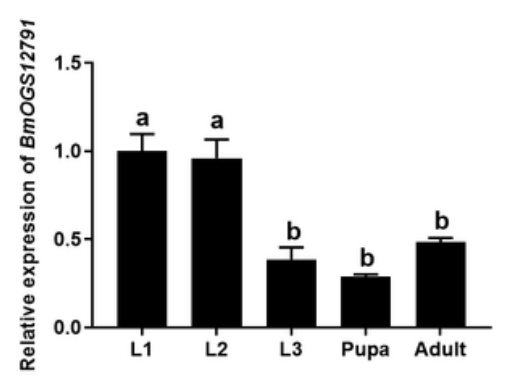

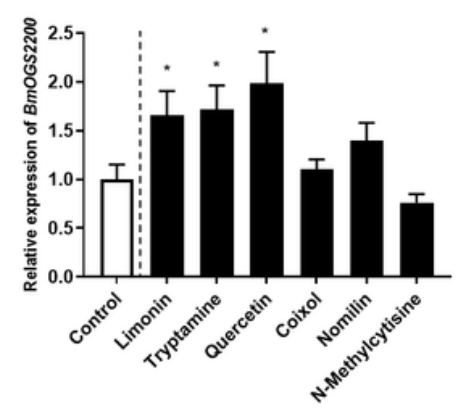

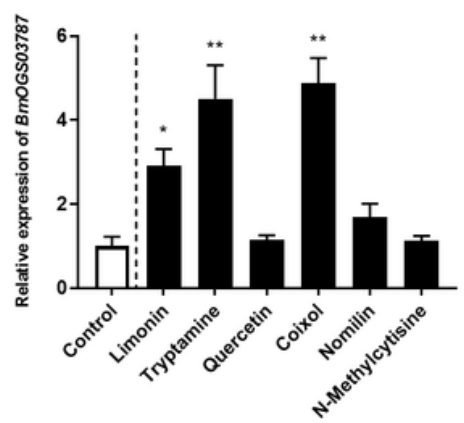

C

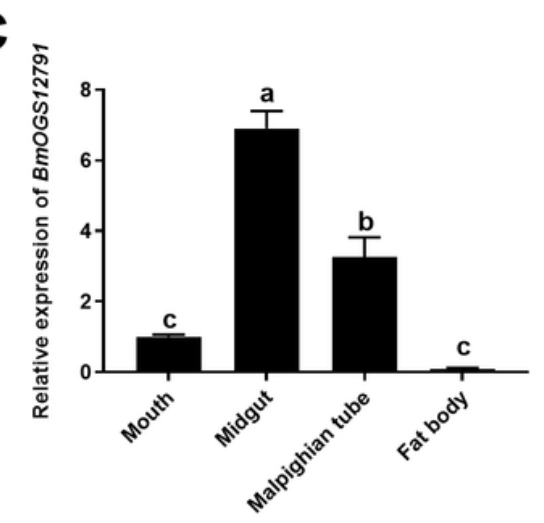

Figure 4

Relative expression of differentially expressed detoxification genes. a: Confirmatory expression profiles of 6 genes screened in the transcriptome of wild-type B. minax by qRT-PCR. ${ }^{*} p<0.05, * \star 0.001<p<0.01$, *** $p<0.001$ (t-test). b: Relative expression levels of BmOGS12791 in different developmental stages of $B$. minax. c: Relative expression of BmOGS12791 in various tissues of 2nd-instar B. minax larvae. Data are presented as the mean \pm SE; different letters denote a significant difference among different samples $(p<$ 0.05 , one-way ANOVA with LSD test). 

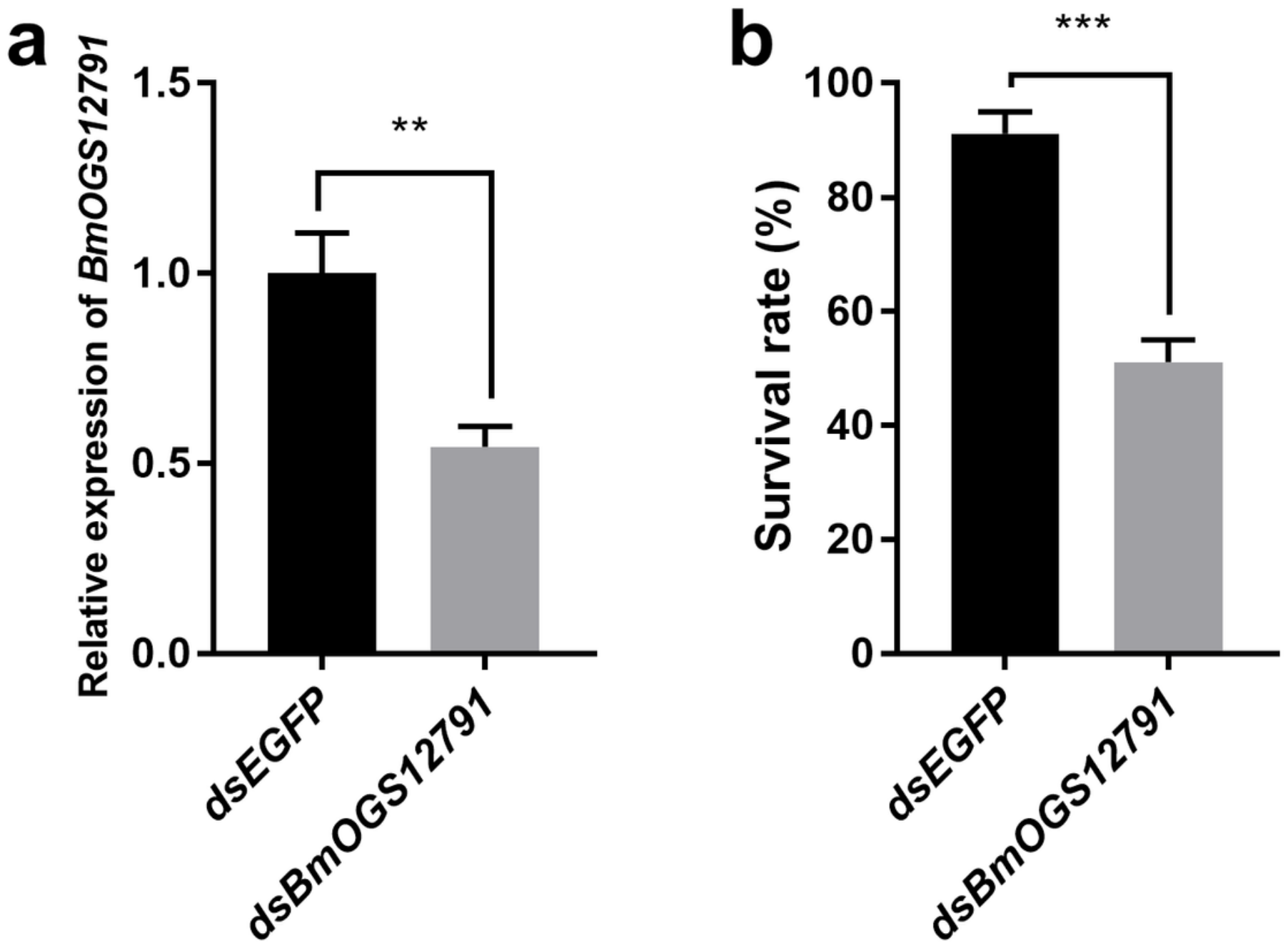

Figure 5

The mean influence of the BmOGS12791 gene on host adaptation in Bactrocera minax. a: Bm0GS12791 mRNA levels of B. minax after dsRNA injection. b: Survival rate of BmOGS12791 knockdown and control B. minax larvae. ${ }^{\star} p<0.05, * \star$ means $0.001<p<0.01, * \star * p<0.001$ (t-test)

\section{Supplementary Files}

This is a list of supplementary files associated with this preprint. Click to download.

- Table1.docx

- Tables1.docx

- Figs1.png

- Figs2.png 
- FigS3.png

- Graphicabstract.png

Page 19/19 